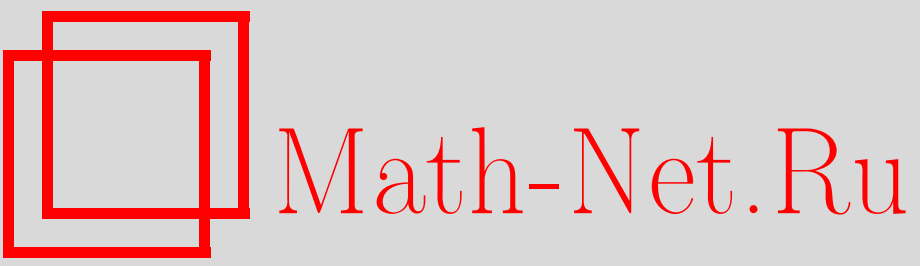

О. Линдблом, Н. Эйлер, Решение уравнений Больцмана для дискретных скоростей при помощи уравнений Бейтмена и Риккати, ТМФ, 2002, том 131, номер 2, 179-193

DOI: https://doi.org/10.4213/tmf322

Использование Общероссийского математического портала Math-Net.Ru подразумевает, что вы прочитали и согласны с пользовательским соглашением

http: //www . mathnet.ru/rus/agreement

Параметры загрузки:

IP : 52.87 .193 .239

26 апреля 2023 г., 12:47:17

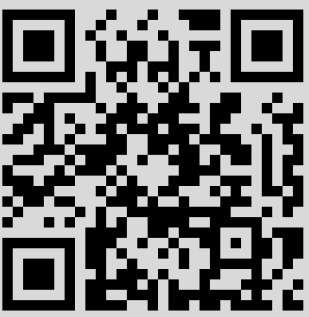




\section{РЕШЕНИЕ УРАВНЕНИЙ БОЛЬЦМАНА ДЛЯ ДИСКРЕТНЫХ СКОРОСТЕЙ ПРИ ПОМОЩИ УРАВНЕНИЙ БЕЙТМЕНА И РИККАТИ}

Предложены несколько подходов к решению двух уравнений Больцмана для дискретных скоростей с помошью анзаца масштабирования и с использованием усеченных разложений Пенлеве. Решения двумерных и трехмерных уравнений Бейтмена для условий многообразия особенностей используются, чтобы свести задачу к уравнениям Риккати. Тест Пенлеве не работает для этих уравнений.

\section{1. ВВЕДЕНИЕ}

Анализ Пенлеве, примененный к дифференциальным уравнениям или системам в частных производных, является полезным тестом на интегрируемость, но дает лишь необходимое условие [1]. Хотя такой анализ (известный как тест Пенлеве) в общем случае представляет собой весьма простую процедуру при его реализации для заданных дифференциальных уравнений или систем в частных производных, он имеет один существенный недостаток, заключаюшийся в том, что результат теста не инвариантен относительно (даже локальных) преобразований уравнения (см., например, книгу Стиба и Эйлера [2]). Для уравнений, которые не проходят тест Пенлеве, можно вывести условия, накладываемые на переменную разложения Пенлеве $\phi,-$ так называемые условия многообразия особенностей, которые могут помочь решить эти уравнения [3]. Эйлер и Линдблом показали [4] для некоторого класса многомерных волновых уравнений, что $n$-мерное уравнение Бейтмена

$$
\operatorname{det}\left(\begin{array}{ccccc}
0 & \phi_{x_{0}} & \phi_{x_{1}} & \ldots & \phi_{x_{n-1}} \\
\phi_{x_{0}} & \phi_{x_{0} x_{0}} & \phi_{x_{0} x_{1}} & \ldots & \phi_{x_{0} x_{n-1}} \\
\phi_{x_{1}} & \phi_{x_{0} x_{1}} & \phi_{x_{1} x_{1}} & \ldots & \phi_{x_{1} x_{n-1}} \\
\cdot & . & \cdot & . & \cdot \\
\cdot & . & . & . & . \\
\cdot & . & \cdot & . & . \\
\phi_{x_{n-1}} & \phi_{x_{0} x_{n-1}} & \phi_{x_{1} x_{n-1}} & \ldots & \phi_{x_{n-1} x_{n-1}}
\end{array}\right)=0
$$

\footnotetext{
*Department of Mathematics Luleå University of Technology, Luleå, Sweden. E-mail: Norbert@sm.luth.se
} 
удовлетворяет условиям многообразия особенностей для некоторых $n$-мерных волновых уравнений. В настоящей работе мы используем этот факт для построения решений двух систем уравнений Больцмана для дискретных скоростей в $(1+1)$ - и $(1+2)$-мерных случаях (вывод этих уравнений и их физическую интерпретацию см. в [5], [6]).

Наш подход аналогичен методу, предложенному Кариэлло и Табором [7], в том смысле, что мы масштабируем усеченное на нулевом уровне разложение Пенлеве некоторой функцией $\phi$. Такая процедура есть не что иное, как анзац масштабирования. Такой подход, вообше говоря, приводит к трудностям, связанным с условиями совместимости. Однако, используя неявные решения уравнений Бейтмена, нам удалось свести эти условия к уравнениям Риккати (см. ниже предложения 1 и 2).

\section{2. (1 + 1)-МЕРНОЕ УРАВНЕНИЕ БОЛЬЦМАНА}

Рассмотрим $(1+1)$-мерную трехскоростную модель, заданную следуюшими уравнениями Больцмана [5]:

$$
\begin{aligned}
\frac{\partial u}{\partial t}+\frac{\partial u}{\partial x} & =\frac{1}{\varepsilon}\left(v^{2}-u w\right) \\
\frac{\partial v}{\partial t} & =-\frac{1}{2 \varepsilon}\left(v^{2}-u w\right) \\
\frac{\partial w}{\partial t}-\frac{\partial w}{\partial x} & =\frac{1}{\varepsilon}\left(v^{2}-u w\right)
\end{aligned}
$$

где $\varepsilon \in \mathbb{R}$ - аналог длины свободного пути.

Для системы (2.1) тест Пенлеве неэффективен, что приводит к некоторому ограничению на многообразие особенностей. Поэтому следует обсудить этот результат более подробно и явно вывести условие многообразия особенностей.

Рассмотрим разложение Пенлеве для системы (2.1):

$$
\begin{aligned}
& u(t, x)=\phi^{-1}(t, x) \sum_{j=0}^{\infty} u_{j}(t, x) \phi^{j}(t, x), \\
& v(t, x)=\phi^{-1}(t, x) \sum_{j=0}^{\infty} v_{j}(t, x) \phi^{j}(t, x), \\
& w(t, x)=\phi^{-1}(t, x) \sum_{j=0}^{\infty} w_{j}(t, x) \phi^{j}(t, x) .
\end{aligned}
$$

В резонансе $r=1$ получаем

$$
\left(\begin{array}{c}
\varepsilon\left(u_{0 t}+u_{0 x}\right) \\
-2 \varepsilon v_{0 t} \\
\varepsilon\left(w_{0 t}-w_{0 x}\right)
\end{array}\right)=\left(\begin{array}{ccc}
-w_{0} & 2 v_{0} & -u_{0} \\
-w_{0} & 2 v_{0} & -u_{0} \\
-w_{0} & 2 v_{0} & -u_{0}
\end{array}\right)\left(\begin{array}{c}
u_{1} \\
v_{1} \\
w_{1}
\end{array}\right)
$$


где

$$
\begin{aligned}
u_{0}(t, x) & =-4 \varepsilon \phi_{t}^{2} \frac{\phi_{x}-\phi_{t}}{\phi_{x}^{2}+3 \phi_{t}^{2}}, \\
v_{0}(t, x) & =2 \varepsilon \phi_{t} \frac{\phi_{x}^{2}-\phi_{t}^{2}}{\phi_{x}^{2}+3 \phi_{t}^{2}}, \\
w_{0}(t, x) & =4 \varepsilon \phi_{t}^{2} \frac{\phi_{x}+\phi_{t}}{\phi_{x}^{2}+3 \phi_{t}^{2}},
\end{aligned}
$$

а $\phi_{x}^{2}+3 \phi_{t}^{2} \neq 0$. Как обычно, нижние индексы обозначают частное дифференцирование. Так как должны выполняться равенства

$$
u_{0 t}+u_{0 x}=-2 v_{0 t}, \quad u_{0 t}+u_{0 x}=w_{0 t}-w_{0 x}
$$

то мы получаем следуюшее ограничение на $\phi$ :

$$
\phi_{t}^{2} \phi_{x x}+\phi_{x}^{2} \phi_{t t}-2 \phi_{x} \phi_{t} \phi_{x t}=0
$$

которое представляет собой так называемое двумерное уравнение Бейтмена [4]. Общее (неявное) решение уравнения (2.5) может быть записано в виде

$$
f(\phi)=x+g(\phi) t
$$

где $f$ и $g$ являются произвольными гладкими функциями от $\phi$. Чтобы резонансные коэффициенты в (2.2) были произвольными, функция $\phi$ должна удовлетворять двумерному уравнению Бейтмена (2.5), которое является условием многообразия особенностей.

Для того чтобы получить некоторые решения уравнений (2.1), нам необходима следуюшая

ЛЕмма 1. Для трехскоростной модели (2.1) усеченное разложение Пенлеве

$$
u(t, x)=\frac{u_{0}}{\phi}, \quad v(t, x)=\frac{v_{0}}{\phi}, \quad w(t, x)=\frac{w_{0}}{\phi},
$$

где $u_{0}, v_{0}$ и $w_{0}$ заданы выражениями (2.3), дает условия (2.5) и

$$
-3 \phi_{t}^{4} \phi_{t t}-6 \phi_{t}^{2} \phi_{x}^{2} \phi_{t t}+\phi_{x}^{4} \phi_{t t}+8 \phi_{t}^{3} \phi_{x} \phi_{t x}=0
$$

с решениями

$$
\phi(t, x)=\frac{x+k_{0} t-b_{2}}{b_{1}},
$$

где $k_{0} \in \mathbb{R} \backslash\{0, \pm 1\}, \quad b_{1} \in \mathbb{R} \backslash\{0\}, \quad b_{2} \in \mathbb{R} ;$

$$
\phi(t, x)=F(x \pm t),
$$

$F$ - прочзвольная обратимая функиия;

$$
\phi(t, x)=\frac{1}{A}\left(\frac{b_{1}-t}{x-b_{2}}+\ln \left(\frac{x+t-b_{1}-b_{2}}{x-t+b_{1}-b_{2}}\right)^{2}+B\right),
$$

əде $\left\{A, b_{1}\right\} \in \mathbb{R} \backslash\{0\} u\left\{b_{1}, B\right\} \in \mathbb{R}$. 
ДокАЗАТЕЛЬСТво содержится в обсуждении приводимых ниже случаев 1-3.

Подставляя решение двумерного уравнения Бейтмена (2.6) в (2.5) и (2.8) и затем приравнивая коэффициенты разложения по переменной $t$ при $t^{0}$ и $t^{1}$ нулю, мы получаем следуюшие условия на $f$ и $g$ :

$$
\begin{gathered}
\frac{d^{2} f}{d \phi^{2}} g\left(g^{2}-1\right)\left(3 g^{2}+1\right)-2 \frac{d f}{d \phi} \frac{d g}{d \phi}\left(g^{2}+1\right)\left(3 g^{2}-1\right)=0, \\
\frac{d^{2} g}{d \phi^{2}} g\left(g^{2}-1\right)\left(3 g^{2}+1\right)-2\left(\frac{d g}{d \phi}\right)^{2}\left(g^{2}+1\right)\left(3 g^{2}-1\right)=0 .
\end{gathered}
$$

Чтобы решить систему $(2.9),(2.10)$, необходимо рассмотреть три случая.

СлучАй 1 . Пусть $g=k_{0}, k_{0} \notin\{0, \pm 1\}$. Уравнение (2.9) имеет решение

$$
f(\phi)=b_{1} \phi+b_{2}, \quad b_{1} \in \mathbb{R} \backslash\{0\}, \quad b_{2} \in \mathbb{R},
$$

которое с учетом (2.6) приводит к соотношению

$$
\phi(t, x)=\frac{x+k_{0} t-b_{2}}{b_{1}} .
$$

Получаем следуюшее решение системы (2.1):

$$
\begin{aligned}
& u(t, x)=\frac{4 \varepsilon k_{0}^{2}\left(k_{0}-1\right)}{3 k_{0}^{2}+1}\left(x+k_{0} t-b_{2}\right)^{-1}, \\
& v(t, x)=-\frac{2 \varepsilon k_{0}\left(k_{0}^{2}-1\right)}{3 k_{0}^{2}+1}\left(x+k_{0} t-b_{2}\right)^{-1}, \\
& w(t, x)=\frac{4 \varepsilon k_{0}^{2}\left(k_{0}+1\right)}{3 k_{0}^{2}+1}\left(x+k_{0} t-b_{2}\right)^{-1} .
\end{aligned}
$$

Заметим, что при $g=0$ мы получаем тривиальное решение $u=v=w=0$ системы (2.1).

СлучАй 2. Пусть $g= \pm 1$. Тогда $\phi_{t}= \pm \phi_{x}$. Из (2.6) следует

$$
\phi(t, x)=F(x \pm t),
$$

где $F$ - некоторая произвольная обратимая функция. Решения системы (2.1) принимают вид

$$
\begin{aligned}
u(t, x) & =0 \\
v(t, x) & =0 \\
w(t, x) & =\frac{2 \varepsilon F^{\prime}(x+t)}{F(x+t)}
\end{aligned}
$$

при $g=1$ и

$$
\begin{aligned}
u(t, x) & =-\frac{2 \varepsilon F^{\prime}(x-t)}{F(x-t)}, \\
v(t, x) & =0, \\
w(t, x) & =0
\end{aligned}
$$

при $g=-1$. 
СлучАй 3 . Пусть $g^{\prime}(\phi) \neq 0$. Система $(2.9),(2.10)$ приводится к виду

$$
\frac{f^{\prime \prime}}{f^{\prime}}=\frac{g^{\prime \prime}}{g^{\prime}} \Leftrightarrow f(\phi)=b_{1} g(\phi)+b_{2},
$$

где $b_{1}$ и $b_{2}$ - постоянные интегрирования, $b_{1} \neq 0$. Теперь $g$ можно выразить через $t$ и $x$ при помоши $(2.6)$ и $(2.13)$ :

$$
g(t, x)=\frac{x-b_{2}}{b_{1}-t} .
$$

Кроме того, из уравнения (2.10) получаем

$$
\frac{d g}{d \phi}=A \frac{g^{2}\left(g^{2}-1\right)}{3 g^{2}+1},
$$

где $A$ - ненулевая постоянная интегрирования. Обшим решением уравнения (2.15) является функция

$$
\phi=\frac{1}{A}\left(\frac{1}{g(\phi)}+\ln \left(\frac{g(\phi)-1}{g(\phi)+1}\right)^{2}+B\right),
$$

( $B$ - постоянная интегрирования), а (2.14) дает $\phi\left(\right.$ с $\left.g^{\prime}(\phi) \neq 0\right)$ в наиболее общем виде:

$$
\phi(t, x)=\frac{1}{A}\left(\frac{b_{1}-t}{x-b_{2}}+\ln \left(\frac{x+t-b_{1}-b_{2}}{x-t+b_{1}-b_{2}}\right)^{2}+B\right) .
$$

Здесь $u_{0}, v_{0}$ и $w_{0}$ были определены в (2.3). Выражения (2.7) вместе с (2.17) теперь приводят к следуюшему решению системы (2.1):

где

$$
\begin{aligned}
u(t, x) & =4 \varepsilon \frac{b_{2}-x}{\left(t-x-b_{1}+b_{2}\right) H(t, x)}, \\
v(t, x) & =-2 \varepsilon \frac{1}{H(t, x)}, \\
w(t, x) & =-4 \varepsilon \frac{b_{2}-x}{\left(t+x-b_{1}-b_{2}\right) H(t, x)},
\end{aligned}
$$

$$
H(t, x)=b_{1}-t+\left(x-b_{2}\right)\left[\ln \left(\frac{x+t-b_{1}-b_{2}}{x-t+b_{1}-b_{2}}\right)^{2}+B\right] .
$$

Докажем следующее

ПРеДЛОЖенИЕ 1. Решения трехскоростной модели (2.1) могут бъть представленьг в виде

$$
u(t, x)=u_{0} H_{1}(\phi), \quad v(t, x)=v_{0} H_{2}(\phi), \quad w(t, x)=w_{0} H_{3}(\phi),
$$

2де

$$
\begin{aligned}
& H_{1}(\phi)=H_{3}(\phi)+k_{1}, \\
& H_{2}(\phi)=H_{3}(\phi)+k_{2}, \quad k_{1}, k_{2} \in \mathbb{R},
\end{aligned}
$$

а $\mathrm{H}_{3}$ удовлетворяет уравнению Риккати

$$
\frac{d H_{3}}{d \phi}=-H_{3}^{2}+2 \frac{k_{2}\left(g^{2}(\phi)-1\right)-2 k_{1} g^{2}(\phi)}{3 g^{2}(\phi)+1} H_{3}+\frac{k_{2}^{2}\left(g^{2}(\phi)-1\right)}{3 g^{2}(\phi)+1} .
$$

Здесь $u_{0}, v_{0}$ и $w_{0}$ задань в $(2.3), g(\phi)=\phi_{t} / \phi_{x}$, а функция $\phi$ должна удовлетворять условиям (2.5) и (2.8). 
ДокАЗАТЕльСтво. Применяя анзац масштабирования

$$
u(t, x)=\frac{u_{0}}{\phi} f_{1}(\phi), \quad v(t, x)=\frac{v_{0}}{\phi} f_{2}(\phi), \quad w(t, x)=\frac{w_{0}}{\phi} f_{3}(\phi)
$$

в $(2.1)$ и используя лемму 1 , приходим к следуюшим условиям на $f_{1}, f_{2}$ и $f_{3}$ :

$$
\begin{aligned}
& \left(3 f_{1}^{\prime} \phi-3 f_{1}-f_{2}^{2}+4 f_{1} f_{3}\right) \phi_{t}^{2}+\left(f_{1}^{\prime} \phi-f_{1}+f_{2}^{2}\right) \phi_{x}^{2}=0, \\
& \left(3 f_{2}^{\prime} \phi-3 f_{2}-f_{2}^{2}+4 f_{1} f_{3}\right) \phi_{t}^{2}+\left(f_{2}^{\prime} \phi-f_{2}+f_{2}^{2}\right) \phi_{x}^{2}=0, \\
& \left(3 f_{3}^{\prime} \phi-3 f_{3}-f_{2}^{2}+4 f_{1} f_{3}\right) \phi_{t}^{2}+\left(f_{3}^{\prime} \phi-f_{3}+f_{2}^{2}\right) \phi_{x}^{2}=0 .
\end{aligned}
$$

При помоши подстановки $f_{i}(\phi)=H_{i}(\phi) \phi, i=1,2,3$, систему $(2.21)$ можно упростить:

$$
\begin{aligned}
& \left(3 H_{1}^{\prime}-H_{2}^{2}+4 H_{1} H_{3}\right) \phi_{t}^{2}+\left(H_{1}^{\prime}+H_{2}^{2}\right) \phi_{x}^{2}=0, \\
& \left(3 H_{2}^{\prime}-H_{2}^{2}+4 H_{1} H_{3}\right) \phi_{t}^{2}+\left(H_{2}^{\prime}+H_{2}^{2}\right) \phi_{x}^{2}=0, \\
& \left(3 H_{3}^{\prime}-H_{2}^{2}+4 H_{1} H_{3}\right) \phi_{t}^{2}+\left(H_{3}^{\prime}+H_{2}^{2}\right) \phi_{x}^{2}=0 .
\end{aligned}
$$

После некоторых алгебраических операций систему (2.22) можно привести к виду

$$
\begin{aligned}
\left(H_{1}^{\prime}-H_{3}^{\prime}\right)\left(\phi_{x}^{2}+3 \phi_{t}^{2}\right) & =0, \\
\left(H_{2}^{\prime}-H_{3}^{\prime}\right)\left(\phi_{x}^{2}+3 \phi_{t}^{2}\right) & =0, \\
\left(3 H_{3}^{\prime}-H_{2}^{2}+4 H_{1} H_{3}\right) \phi_{t}^{2}+\left(H_{3}^{\prime}+H_{2}^{2}\right) \phi_{x}^{2} & =0 .
\end{aligned}
$$

В результате дифференцирования решения (2.6) по $t$ и $x$ получим

$$
\frac{\phi_{t}}{\phi_{x}}=g(\phi) \text {. }
$$

Таким образом, справедливы соотношения (2.19), которые после подстановки в последнее уравнение системы (2.23) и использования (2.24) дают уравнение Риккати (2.20).

ЗАмечАние 1 . В случае $H_{1}=H_{2}=H_{3}$ получаем разложение Пенлеве (2.7).

Рассмотрим несколько примеров применения предложения 1.

ПримеР 1 . При $g(\phi)=2, k_{1}=1$ и $k_{2}=2$ уравнение (2.20) упрошается:

$$
H_{3}^{\prime}=-H_{3}^{2}-\frac{4}{13} H_{3}+\frac{12}{13}
$$

оно имеет общее решение

$$
H_{3}(\phi)=-\frac{2}{13}+\frac{4}{13} \sqrt{10} \operatorname{th}\left(\frac{4}{13} \sqrt{10} \phi+C\right),
$$

где $C$ - постоянная интегрирования. Ввиду (2.6) и (2.11) $\phi=x+2 t$. Поэтому получаем следуюшее решение уравнений (2.1):

$$
\begin{aligned}
& u(t, x)=u_{0}\left(H_{3}(\phi)+1\right)=\frac{16 \varepsilon}{13}\left(\frac{11}{13}+\frac{4}{13} \sqrt{10} \operatorname{th}\left(\frac{4}{13} \sqrt{10}(x+2 t)+C\right)\right), \\
& v(t, x)=v_{0}\left(H_{3}(\phi)+2\right)=-\frac{12 \varepsilon}{13}\left(\frac{24}{13}+\frac{4}{13} \sqrt{10} \operatorname{th}\left(\frac{4}{13} \sqrt{10}(x+2 t)+C\right)\right), \\
& w(t, x)=w_{0} H_{3}(\phi)=\frac{48 \varepsilon}{13}\left(-\frac{2}{13}+\frac{4}{13} \sqrt{10} \operatorname{th}\left(\frac{4}{13} \sqrt{10}(x+2 t)+C\right)\right) .
\end{aligned}
$$


ЗАмечАниЕ 2. Пример 1 относится к случаю 1 при $k_{0}=2, b_{1}=1$ и $b_{2}=0$.

ПримеР 2. При $g(\phi)= \pm 1$ уравнение (2.20) упрощается:

$$
H_{3}^{\prime}=-H_{3}^{2}-k_{1} H_{3}
$$

оно имеет общее решение

$$
H_{3}(\phi)=\frac{k_{1}}{C k_{1} e^{k_{1} \phi}-1},
$$

где $C$-постоянная интегрирования. С учетом (2.12) решения системы (2.1) принимают вид

$$
\begin{aligned}
u(t, x) & =0, \\
v(t, x) & =0, \\
w(t, x) & =\frac{2 \varepsilon k_{1} F^{\prime}(x+t)}{C k_{1} e^{k_{1} F(x+t)}-1}
\end{aligned}
$$

при $g=1$ и

$$
\begin{aligned}
u(t, x) & =\frac{2 \varepsilon k_{1} F^{\prime}(x-t)}{C k_{1} e^{k_{1} F(x-t)}-1}, \\
v(t, x) & =0 \\
w(t, x) & =0
\end{aligned}
$$

при $g=-1$.

ПримеР 3. При $g^{\prime}(\phi) \neq 0$ (см. случай 3$)$ после подстановки $H_{3}(\phi)=\widetilde{H}_{3}(g)$ и использования соотношения (2.15) уравнение Риккати (2.20) принимает вид

$$
A \frac{d \widetilde{H}_{3}}{d g}=-\frac{3 g^{2}+1}{g^{2}\left(g^{2}-1\right)} \widetilde{H}_{3}^{2}+2 \frac{k_{2}\left(g^{2}-1\right)-2 k_{1} g^{2}}{g^{2}\left(g^{2}-1\right)} \widetilde{H}_{3}+\frac{k_{2}^{2}}{g^{2}} .
$$

При $k_{2}=0$ и $k_{1} / A=n \neq 0$ уравнение (2.25) допускает общее решение

$$
\widetilde{H}_{3}(g)=A\left(\frac{g+1}{g-1}\right)^{2 n}\left(-\frac{3}{4 n}\left(\frac{g+1}{g-1}\right)^{2 n}+\int\left(\frac{g+1}{g-1}\right)^{2 n} \frac{1}{g^{2}\left(g^{2}-1\right)} d g+C\right)^{-1},
$$

где $C$ - постоянная интегрирования. Если $n \neq 0$ - некоторое целое число, то решение (2.26) можно представить в элементарных функциях. Например, выбор $n=2$ дает решение (2.26) в замкнутой форме:

$$
\widetilde{H}_{3}(g)=3 A g(g+1)^{4}\left(3 g(g-1)^{4}\left(A C+8 \ln \left|\frac{g-1}{g}\right|\right)+15 g^{4}-84 g^{3}+94 g^{2}-52 g+3\right)^{-1} .
$$

Можно также рассматривать рациональные $n$. Например, выбор $n=1 / 4$ дает

$$
\widetilde{H}_{3}(g)=A g\left(g^{2}-1\right)^{\frac{1}{2}}\left[g(g-1)\left(\operatorname{arctg}\left(g^{2}-1\right)^{-\frac{1}{2}}+C\right)+(1-5 g)\left(g^{2}-1\right)^{\frac{1}{2}}\right]^{-1} .
$$


Решения уравнений (2.1) тогда получаем при помоши (2.18), где $u_{0}, v_{0}$ и $w_{0}$ определены в $(2.3)$, а $\phi-$ в $(2.17)$ :

$$
\begin{aligned}
u(t, x) & =\frac{4 \varepsilon}{A\left(x-t+b_{1}-b_{2}\right)}\left(\widetilde{H}_{3}(g)+n A\right), \\
v(t, x) & =\frac{2 \varepsilon}{A\left(b_{2}-x\right)} \widetilde{H}_{3}(g), \\
w(t, x) & =\frac{4 \varepsilon}{A\left(x+t-b_{1}-b_{2}\right)} \widetilde{H}_{3}(g) .
\end{aligned}
$$

Здесь $\widetilde{H}_{3}(g)$ задано в $(2.25)$, а $g-$ в $(2.14)$.

\section{3. (1 + 2)-МЕРНОЕ УРАВНЕНИЕ БОЛЬЦМАНА}

Теперь рассмотрим $(1+2)$-мерную четырехскоростную модель, заданную следующими уравнениями Больцмана [5]:

$$
\begin{array}{lll}
\frac{\partial u}{\partial t}+c \frac{\partial u}{\partial x}=\sigma(v \tilde{v}-u \tilde{u}), & \frac{\partial v}{\partial t}+c \frac{\partial v}{\partial y}=\sigma(u \tilde{u}-v \tilde{v}), \\
\frac{\partial \tilde{u}}{\partial t}-c \frac{\partial \tilde{u}}{\partial x}=\sigma(v \tilde{v}-u \tilde{u}), & \frac{\partial \tilde{v}}{\partial t}-c \frac{\partial \tilde{v}}{\partial y}=\sigma(u \tilde{u}-v \tilde{v}),
\end{array}
$$

где $c, \sigma \in \mathbb{R} \backslash\{0\}$, а $u, \tilde{u}, v$ и $\tilde{v}$ - некоторые функции от $t, x$ и $y$. Система (3.1) не поддается тесту Пенлеве, что приводит к ограничению на многообразие особенностей. Обсудим этот результат более подробно и получим явное условие многообразия особенностей.

Система (3.1) допускает разложения

$$
\begin{aligned}
& u(t, x, y)=\phi^{-1}(t, x, y) \sum_{j=0}^{\infty} u_{j}(t, x, y) \phi^{j}(t, x, y), \\
& \tilde{u}(t, x, y)=\phi^{-1}(t, x, y) \sum_{j=0}^{\infty} \tilde{u}_{j}(t, x, y) \phi^{j}(t, x, y), \\
& v(t, x, y)=\phi^{-1}(t, x, y) \sum_{j=0}^{\infty} v_{j}(t, x, y) \phi^{j}(t, x, y), \\
& \tilde{v}(t, x, y)=\phi^{-1}(t, x, y) \sum_{j=0}^{\infty} \tilde{v}_{j}(t, x, y) \phi^{j}(t, x, y) .
\end{aligned}
$$

В резонансе $r=1$ имеем

$$
\frac{1}{\sigma}\left(\begin{array}{c}
u_{0 t}+c u_{0 x} \\
\tilde{u}_{0 t}-c \tilde{u}_{0 x} \\
-\left(v_{0 t}+c v_{0 y}\right) \\
-\left(\tilde{v}_{0 t}-c \tilde{v}_{0 y}\right)
\end{array}\right)=\left(\begin{array}{cccc}
-\tilde{u}_{0} & -u_{0} & \tilde{v}_{0} & v_{0} \\
-\tilde{u}_{0} & -u_{0} & \tilde{v}_{0} & v_{0} \\
-\tilde{u}_{0} & -u_{0} & \tilde{v}_{0} & v_{0} \\
-\tilde{u}_{0} & -u_{0} & \tilde{v}_{0} & v_{0}
\end{array}\right)\left(\begin{array}{c}
u_{1} \\
\tilde{u}_{1} \\
v_{1} \\
\tilde{v}_{1}
\end{array}\right)
$$


где $u_{0}, \tilde{u}_{0}, v_{0}$ и $\tilde{v}_{0}$ задаются следующими выражениями:

$$
\begin{aligned}
& u_{0}(t, x, y)=\frac{1}{c^{2} \sigma} \frac{\left(\phi_{t}-c \phi_{x}\right)\left(\phi_{t}^{2}-c^{2} \phi_{y}^{2}\right)}{\phi_{x}^{2}-\phi_{y}^{2}}, \\
& \tilde{u}_{0}(t, x, y)=\frac{1}{c^{2} \sigma} \frac{\left(\phi_{t}+c \phi_{x}\right)\left(\phi_{t}^{2}-c^{2} \phi_{y}^{2}\right)}{\phi_{x}^{2}-\phi_{y}^{2}}, \\
& v_{0}(t, x, y)=-\frac{1}{c^{2} \sigma} \frac{\left(\phi_{t}-c \phi_{y}\right)\left(\phi_{t}^{2}-c^{2} \phi_{x}^{2}\right)}{\phi_{x}^{2}-\phi_{y}^{2}}, \\
& \tilde{v}_{0}(t, x, y)=-\frac{1}{c^{2} \sigma} \frac{\left(\phi_{t}+c \phi_{y}\right)\left(\phi_{t}^{2}-c^{2} \phi_{x}^{2}\right)}{\phi_{x}^{2}-\phi_{y}^{2}}
\end{aligned}
$$

причем $\phi_{x}^{2}-\phi_{y}^{2} \neq 0$. Требования

$$
u_{0 t}+c u_{0 x}=\tilde{u}_{0 t}-c \tilde{u}_{0 x}, \quad u_{0 t}+c u_{0 x}=-v_{0 t}+c v_{0 y}, \quad v_{0 t}+c v_{0 y}=\tilde{v}_{0 t}+c \tilde{v}_{0 y}
$$

приводят к условиям многообразия особенностей

$$
\begin{aligned}
\phi_{t} \phi_{x} \phi_{t t} & \left(\phi_{y}^{2}-\phi_{x}^{2}\right)+\phi_{t x}\left[2 \phi_{x}^{2} \phi_{t}^{2}-\phi_{y}^{2}\left(c^{2} \phi_{x}^{2}+\phi_{t}^{2}\right)\right]+ \\
& +\phi_{t} \phi_{x} \phi_{x x}\left(c^{2} \phi_{y}^{2}-\phi_{t}^{2}\right)+\phi_{y}\left(c^{2} \phi_{x}^{2}-\phi_{t}^{2}\right)\left(\phi_{x} \phi_{t y}-\phi_{t} \phi_{x y}\right)=0 \\
\phi_{t} \phi_{y} \phi_{t t} & \left(\phi_{x}^{2}-\phi_{y}^{2}\right)+\phi_{t y}\left[2 \phi_{t}^{2} \phi_{y}^{2}-\phi_{x}^{2}\left(c^{2} \phi_{y}^{2}+\phi_{t}^{2}\right)\right]+ \\
& +\phi_{t} \phi_{y} \phi_{y y}\left(c^{2} \phi_{x}^{2}-\phi_{t}^{2}\right)+\phi_{x}\left(c^{2} \phi_{y}^{2}-\phi_{t}^{2}\right)\left(\phi_{y} \phi_{t x}-\phi_{t} \phi_{x y}\right)=0 \\
\phi_{t t}\left(\phi_{x}\right. & \left.+\phi_{y}\right)\left(\phi_{x}-\phi_{y}\right)^{2}\left[c\left(\phi_{x}+\phi_{y}\right)-2 \phi_{t}\right]+\phi_{x x}\left(c^{2} \phi_{y}^{2}-\phi_{t}^{2}\right)\left[c\left(\phi_{x}^{2}+\phi_{y}^{2}\right)-2 \phi_{t} \phi_{x}\right]- \\
& -\phi_{y y}\left(c^{2} \phi_{x}^{2}-\phi_{t}^{2}\right)\left[c\left(\phi_{x}^{2}+\phi_{y}^{2}\right)-2 \phi_{t} \phi_{y}\right]+ \\
& +2 \phi_{t x}\left(\phi_{y}-\phi_{x}\right)\left[c \phi_{t} \phi_{x}\left(\phi_{x}+\phi_{y}\right)-\phi_{t}^{2}\left(\phi_{y}+2 \phi_{x}\right)+c^{2} \phi_{x} \phi_{y}^{2}\right]+ \\
& +2 \phi_{t y}\left(\phi_{x}-\phi_{y}\right)\left[c \phi_{t} \phi_{y}\left(\phi_{x}+\phi_{y}\right)-\phi_{t}^{2}\left(\phi_{x}+2 \phi_{y}\right)+c^{2} \phi_{y} \phi_{x}^{2}\right]+ \\
& +2 \phi_{x y}\left[c \phi_{x} \phi_{y}\left(c^{2}\left(\phi_{x}^{2}+\phi_{y}^{2}\right)-2 \phi_{t}^{2}\right)+\phi_{t}\left(\phi_{x}+\phi_{y}\right)\left(\phi_{t}^{2}-c^{2} \phi_{x} \phi_{y}\right)\right]=0
\end{aligned}
$$

Формула

$$
t f(\phi)+x g(\phi)+y h(\phi)=c_{1}
$$

где $f, g$ и $h$ - некоторые произвольные гладкие функции, удовлетворяет условиям (3.5)-(3.7). Выражение (3.8) является (неявным) решением трехмерного уравнения Бейтмена [4]

$$
\operatorname{det}\left(\begin{array}{cccc}
0 & \phi_{t} & \phi_{x} & \phi_{y} \\
\phi_{t} & \phi_{t t} & \phi_{t x} & \phi_{t y} \\
\phi_{x} & \phi_{t x} & \phi_{x x} & \phi_{x y} \\
\phi_{y} & \phi_{t y} & \phi_{x x} & \phi_{y y}
\end{array}\right)=0
$$

Таким образом, чтобы резонансные коэффициенты в (3.2) были произвольными, достаточно, чтобы функция $\phi$ удовлетворяла трехмерному уравнению Бейтмена (3.9).

Для того чтобы получить решения системы (3.1), нам необходима 
ЛЕмма 2. Для четырехскоростной модели (3.1) усеченный анзаи,

$$
\begin{array}{ll}
u(t, x, y)=u_{0} \phi^{-1}, & v(t, x, y)=v_{0} \phi^{-1} \\
\tilde{u}(t, x, y)=\tilde{u}_{0} \phi^{-1}, & \tilde{v}(t, x, y)=\tilde{v}_{0} \phi^{-1}
\end{array}
$$

где $u_{0}, \tilde{u}_{0}, v_{0} u \tilde{v}_{0}$ определень в (3.3), задает следующее условие на $\phi:$

$$
\left(\frac{\partial}{\partial t}+c \frac{\partial}{\partial x}\right) \frac{\left(\phi_{t}-c \phi_{x}\right)\left(\phi_{t}^{2}-c^{2} \phi_{y}^{2}\right)}{\phi_{x}^{2}-\phi_{y}^{2}}=0
$$

при этом функция ф долэсна удовлетворять условиям (3.5)-(3.7). Решения ф задаются в виде

$$
\phi(t, x, y)=\frac{t+x+a y-b_{2}}{b_{1}}
$$

где $a \in \mathbb{R} \backslash\{ \pm 1\}, \quad b_{1} \in \mathbb{R} \backslash\{0\}$ u $b_{2} \in \mathbb{R}$

$$
\begin{aligned}
\phi(t, x, y)=\frac{1}{A}( & \frac{t+x}{c^{2}\left(c_{1}-c_{2} y\right)}+ \\
& \left.\quad+\frac{1}{2 c c_{2}}\left(1-\frac{1}{c^{2}}\right) \ln \left|\frac{c_{1}+c_{2} c(t+x)-c_{2} y}{c_{1}-c_{2} c(t+x)-c_{2} y}\right|+B\right),
\end{aligned}
$$

əде $\left\{c_{1}, B\right\} \in \mathbb{R}, \quad\left\{c, c_{2}, A\right\} \in \mathbb{R} \backslash\{0\}$.

ДоКАЗАТЕЛЬСТво содержится в обсуждении приведенных ниже случаев $1^{\prime}$ и $2^{\prime}$.

Если подставить (3.8) в (3.11) и приравнять коэфриициенты при $t, x$ и $y$ нулю, то получим следующие условия на $f, g$ и $h$ :

$$
\begin{aligned}
& \alpha f^{\prime \prime}+\beta_{1}\left(f^{\prime}\right)^{2}+\left(\beta_{2} g^{\prime}+\beta_{3} h^{\prime}\right) f^{\prime}=0, \\
& \alpha g^{\prime \prime}+\beta_{2}\left(g^{\prime}\right)^{2}+\left(\beta_{3} h^{\prime}+\beta_{1} f^{\prime}\right) g^{\prime}=0 \\
& \alpha h^{\prime \prime}+\beta_{3}\left(h^{\prime}\right)^{2}+\left(\beta_{1} f^{\prime}+\beta_{2} g^{\prime}\right) h^{\prime}=0
\end{aligned}
$$

где

$$
\begin{aligned}
\alpha & =-\left(g^{2}-h^{2}\right)\left(c^{2} h^{2}-f^{2}\right)\left(c^{2} g^{2}-f^{2}\right), \\
\beta_{1} & =-2 f\left(g^{2}-h^{2}\right)\left(c^{2} g^{2}-2 f^{2}+c^{2} h^{2}\right), \\
\beta_{2} & =-2 g\left(c^{2} h^{2}-f^{2}\right)^{2}, \quad \beta_{3}=2 h\left(c^{2} g^{2}-f^{2}\right)^{2} .
\end{aligned}
$$

Напомним, что $c$ является произвольной постоянной в системе (3.1).

ЗАМЕчАнИЕ 3 . Если функции $f, g$ и $h$ являются константами, то они удовлетворяют системе (3.12), но дают $\phi_{x}^{2}=\phi_{y}^{2}$, что недопустимо (см. (3.3)). Это также справедливо и в случае, когда $g^{2}=h^{2}$, а $f$-произвольная функция. 
СлучАй $1^{\prime}$. Рассмотрим ограничение, когда функции $f$ и $g$ не являются константами и $f(\phi)=g(\phi)$, a $h(\phi)=a g(\phi)$, где $a \neq \pm 1$ (см. замечание 4). Согласно (3.8)

$$
g(\phi)=\frac{c_{1}}{t+x+a y}, \quad c_{1} \in \mathbb{R} .
$$

Кроме того, условия (3.12) приводят к уравнению

$$
g \frac{d^{2} g}{d \phi^{2}}=2\left(\frac{d g}{d \phi}\right)^{2}
$$

решением которого является

$$
g(\phi)=\frac{1}{b_{1} \phi+b_{2}}, \quad b_{1} \in \mathbb{R} \backslash\{0\}, \quad b_{2} \in \mathbb{R},
$$

а соотношение (3.13) дает

$$
\phi(t, x, y)=\frac{t+x+a y-b_{2}}{b_{1}} .
$$

Здесь $u_{0}, \tilde{u}_{0}, v_{0}$ и $\tilde{v}_{0}$ задаются в (3.3). Анзац (3.10) обеспечивает следуюшее решение системы (3.1):

$$
\begin{array}{ll}
u(t, x, y)=\frac{(1-c)\left(c^{2} a^{2}-1\right)}{F(t, x, y)}, & \tilde{u}(t, x, y)=\frac{(1+c)\left(c^{2} a^{2}-1\right)}{F(t, x, y)}, \\
v(t, x, y)=\frac{(c a-1)\left(c^{2}-1\right)}{F(t, x, y)}, & \tilde{v}(t, x, y)=\frac{(c a-1)\left(1-c^{2}\right)}{F(t, x, y)},
\end{array}
$$

где

$$
F(t, x, y)=\sigma c^{2}\left(a^{2}-1\right)\left(t+x+a y-b_{2}\right) .
$$

СлучАй $2^{\prime}$. Пусть функции $f$ и $g$ не являются константами и $f(\phi)=g(\phi)$, а $h(\phi)=$ $c_{2}$. Согласно (3.8)

$$
g(\phi)=\frac{c_{1}-c_{2} y}{t+x}, \quad c_{2} \in \mathbb{R} \backslash\{0\}, \quad c_{1} \in \mathbb{R} .
$$

Кроме того, условия (3.12) приводят к уравнению

$$
\frac{d g}{d \phi}=-A \frac{g^{2}\left(g^{2}-c^{2} c_{2}^{2}\right)}{\left(g^{2}-c_{2}^{2}\right)},
$$

где $A$ - ненулевая постоянная интегрирования. Обшее решение дифференциального уравнения (3.15) имеет вид

$$
\phi(g)=\frac{1}{A}\left(\frac{1}{c^{2} g}+\frac{1}{2 c c_{2}}\left(1-\frac{1}{c^{2}}\right) \ln \left|\frac{g+c c_{2}}{g-c c_{2}}\right|+B\right)
$$


( $B$ - некоторая постоянная интегрирования), и соотношение (3.14) дает

$$
\begin{aligned}
\phi(t, x, y)=\frac{1}{A}( & \frac{t+x}{c^{2}\left(c_{1}-c_{2} y\right)}+ \\
& \left.+\frac{1}{2 c c_{2}}\left(1-\frac{1}{c^{2}}\right) \ln \left|\frac{c_{1}+c_{2} c(t+x)-c_{2} y}{c_{1}-c_{2} c(t+x)-c_{2} y}\right|+B\right) .
\end{aligned}
$$

Здесь $u_{0}, \tilde{u}_{0}, v_{0}$ и $\tilde{v}_{0}$ задаются в (3.3). Анзац (3.10) дает следуюшее решение системы (3.1):

$$
\begin{aligned}
& u(t, x, y)=\frac{\mu(1-c)}{F(t, x, y)}, \quad \tilde{u}(t, x, y)=\frac{\mu(c+1)}{F(t, x, y)} \\
& v(t, x, y)=\frac{\mu\left(c^{2}-1\right)\left(c_{1}-c_{2} y\right)}{\left(c_{1}+c_{2} c(t+x)-c_{2} y\right) F(t, x, y)} \\
& \tilde{v}(t, x, y)=\frac{\mu\left(c^{2}-1\right)\left(c_{1}-c_{2} y\right)}{\left(c_{1}-c_{2} c(t+x)-c_{2} y\right) F(t, x, y)}
\end{aligned}
$$

где $\mu=2 c_{2} c / \sigma$,

$$
F(t, x, y)=2 c_{2} c(t+x)+\left(c_{1}-c_{2} y\right)\left[\left(c^{2}-1\right) \ln \left|\frac{c_{1}+c_{2} c(t+x)-c_{2} y}{c_{1}-c_{2} c(t+x)-c_{2} y}\right|+2 B c^{3} c_{2}\right]
$$

Напомним, что $c_{1}$ является произвольной постоянной в решении трехмерного уравнения Бейтмена (3.8).

ПРЕДЛОЖЕНИЕ 2. Решения четырехскоростной модели (3.1) могут быть записаныл в виде

$$
\begin{array}{ll}
u(t, x, y)=u_{0} H_{1}(\phi), & \tilde{u}(t, x, y)=\tilde{u}_{0} H_{2}(\phi), \\
v(t, x, y)=v_{0} H_{3}(\phi), & \tilde{v}(t, x, y)=\tilde{v}_{0} H_{4}(\phi),
\end{array}
$$

$2 \partial e$

$$
\begin{array}{ll}
H_{1}(\phi)=H_{4}(\phi)+k_{1}, & H_{2}(\phi)=H_{4}(\phi)+k_{2}, \\
H_{3}(\phi)=H_{4}(\phi)+k_{3}, & k_{1}, k_{2}, k_{3} \in \mathbb{R}
\end{array}
$$

а $\mathrm{H}_{4}$ удовлетворяет уравнению Риккати

$$
\begin{aligned}
\frac{d H_{4}}{d \phi}=- & H_{4}^{2}+\frac{k_{1}\left(f^{2}-c^{2} h^{2}\right)+k_{2}\left(f^{2}-c^{2} h^{2}\right)-k_{3}\left(f^{2}-c^{2} g^{2}\right)}{c^{2}\left(h^{2}-g^{2}\right)} H_{4}+ \\
& +\frac{k_{1} k_{2}\left(f^{2}-c^{2} h^{2}\right)}{c^{2}\left(h^{2}-g^{2}\right)}
\end{aligned}
$$

Здесь $u_{0}, \tilde{u}_{0}, v_{0}$ и $\tilde{v}_{0}$ задаются при помощи (3.3), а ф удовлетворяет условию (3.11) и трехмерному уравнению Бейтмена (3.9). 
ДокАЗАТЕЛЬСтво. С помощью анзаца масштабирования

$$
\begin{array}{ll}
u(t, x, y)=\frac{u_{0}}{\phi} f_{1}(\phi), & \tilde{u}(t, x, y)=\frac{\tilde{u}_{0}}{\phi} f_{2}(\phi), \\
v(t, x, y)=\frac{v_{0}}{\phi} f_{3}(\phi), & \tilde{v}(t, x, y)=\frac{\tilde{v}_{0}}{\phi} f_{4}(\phi)
\end{array}
$$

для системы (3.1) и леммы 2 получаем следуюшие условия на $f_{1}, f_{2}, f_{3}$ и $f_{4}$ :

$$
\begin{aligned}
& \left(f_{1} f_{3}-f_{2} f_{4}\right) \phi_{t}^{2}+\left[\left(f_{1}^{\prime} \phi-f_{1}+f_{3} f_{4}\right) \phi_{x}^{2}-\left(f_{1}^{\prime} \phi-f_{1}+f_{1} f_{2}\right) \phi_{y}^{2}\right] c^{2}=0 \\
& \left(f_{1} f_{3}-f_{2} f_{4}\right) \phi_{t}^{2}+\left[\left(f_{2}^{\prime} \phi-f_{2}+f_{3} f_{4}\right) \phi_{x}^{2}-\left(f_{2}^{\prime} \phi-f_{2}+f_{1} f_{2}\right) \phi_{y}^{2}\right] c^{2}=0 \\
& \left(f_{1} f_{3}-f_{2} f_{4}\right) \phi_{t}^{2}+\left[\left(f_{3}^{\prime} \phi-f_{3}+f_{3} f_{4}\right) \phi_{x}^{2}-\left(f_{3}^{\prime} \phi-f_{3}+f_{1} f_{2}\right) \phi_{y}^{2}\right] c^{2}=0 \\
& \left(f_{1} f_{3}-f_{2} f_{4}\right) \phi_{t}^{2}+\left[\left(f_{4}^{\prime} \phi-f_{4}+f_{3} f_{4}\right) \phi_{x}^{2}-\left(f_{4}^{\prime} \phi-f_{4}+f_{1} f_{2}\right) \phi_{y}^{2}\right] c^{2}=0 .
\end{aligned}
$$

Подстановка $f_{i}(\phi)=H_{i}(\phi) \phi, i=1,2,3,4$, упрошает систему $(3.20)$ :

$$
\begin{aligned}
& \left(H_{1} H_{3}-H_{2} H_{4}\right) \phi_{t}^{2}+\left[\left(H_{1}^{\prime}+H_{3} H_{4}\right) \phi_{x}^{2}-\left(H_{1}^{\prime}+H_{1} H_{2}\right) \phi_{y}^{2}\right] c^{2}=0, \\
& \left(H_{1} H_{3}-H_{2} H_{4}\right) \phi_{t}^{2}+\left[\left(H_{2}^{\prime}+H_{3} H_{4}\right) \phi_{x}^{2}-\left(H_{2}^{\prime}+H_{1} H_{2}\right) \phi_{y}^{2}\right] c^{2}=0, \\
& \left(H_{1} H_{3}-H_{2} H_{4}\right) \phi_{t}^{2}+\left[\left(H_{3}^{\prime}+H_{3} H_{4}\right) \phi_{x}^{2}-\left(H_{3}^{\prime}+H_{1} H_{2}\right) \phi_{y}^{2}\right] c^{2}=0, \\
& \left(H_{1} H_{3}-H_{2} H_{4}\right) \phi_{t}^{2}+\left[\left(H_{4}^{\prime}+H_{3} H_{4}\right) \phi_{x}^{2}-\left(H_{4}^{\prime}+H_{1} H_{2}\right) \phi_{y}^{2}\right] c^{2}=0 .
\end{aligned}
$$

При помощи алгебраических преобразований систему (3.21) можно привести к виду

$$
\begin{aligned}
& \left(H_{1}^{\prime}-H_{4}^{\prime}\right)\left(\phi_{x}^{2}-\phi_{y}^{2}\right) c^{2}=0, \\
& \left(H_{2}^{\prime}-H_{4}^{\prime}\right)\left(\phi_{x}^{2}-\phi_{y}^{2}\right) c^{2}=0, \\
& \left(H_{3}^{\prime}-H_{4}^{\prime}\right)\left(\phi_{x}^{2}-\phi_{y}^{2}\right) c^{2}=0, \\
\left(H_{1} H_{3}-H_{2} H_{4}\right) \phi_{t}^{2} & +\left(H_{4}^{\prime}+H_{3} H_{4}\right) c^{2} \phi_{x}^{2}-\left(H_{4}^{\prime}+H_{1} H_{2}\right) c^{2} \phi_{y}^{2}=0 .
\end{aligned}
$$

Дифференцируя (3.8) по $t, x$ и $y$, получим соответственно

$$
\begin{aligned}
\phi_{t} & =-\frac{f(\phi)}{t f^{\prime}(\phi)+x g^{\prime}(\phi)+y h^{\prime}(\phi)}, \\
\phi_{x} & =-\frac{g(\phi)}{t f^{\prime}(\phi)+x g^{\prime}(\phi)+y h^{\prime}(\phi)}, \\
\phi_{y} & =-\frac{h(\phi)}{t f^{\prime}(\phi)+x g^{\prime}(\phi)+y h^{\prime}(\phi)} .
\end{aligned}
$$

Так как $\phi_{x}^{2}-\phi_{y}^{2} \neq 0$, из $(3.22)$ и $(3.23)$ получаем

$$
H_{1}(\phi)=H_{4}(\phi)+k_{1}, \quad H_{2}(\phi)=H_{4}(\phi)+k_{2}, \quad H_{3}(\phi)=H_{4}(\phi)+k_{3},
$$

а уравнение Риккати (3.19) задает условие на $H_{4}$.

ЗАмечАнИЕ 4 . В случае $H_{1}=H_{2}=H_{3}=H_{4}$ имеем анзац (3.10). 
ПримеР 4. Рассмотрим случай, когда функции $f$ и $g$ не являются константами и $f(\phi)=g(\phi)$, а $h(\phi)=c_{2}$, как в примере 3 . В результате подстановки $H_{4}(\phi)=\widetilde{H}_{4}(g)$ и использования (3.15) уравнение Риккати (3.19) преобразуется к виду

$$
\begin{aligned}
A \frac{d \widetilde{H}_{4}}{d g}= & \frac{g^{2}-c_{2}^{2}}{g^{2}\left(g^{2}-c_{2}^{2} c^{2}\right)} \widetilde{H}_{4}^{2}+ \\
& +\frac{g^{2}\left(k_{3}\left(c^{2}-1\right)+k_{1}+k_{2}\right)-c_{2}^{2} c^{2}\left(k_{1}+k_{2}\right)}{c^{2} g^{2}\left(g^{2}-c_{2}^{2} c^{2}\right)} \widetilde{H}_{4}+\frac{k_{1} k_{2}}{c^{2} g^{2}}
\end{aligned}
$$

где $A$ является ненулевой произвольной константой. При $k_{2}=0$ уравнение $(3.24)$ допускает решение

$$
\widetilde{H}_{4}(g)=\frac{A\left(\frac{c_{2} c+g}{c_{2} c-g}\right)^{\lambda} e^{-\frac{k_{1} k_{3}}{g A c^{2}}}}{\int \frac{1}{g^{2}}\left(\frac{g^{2}-c_{2}^{2}}{c_{2}^{2} c^{2}-g^{2}}\right)\left(\frac{c_{2} c+g}{c_{2} c-g}\right)^{\lambda} e^{-\frac{k_{1} k_{3}}{g A c^{2}}} d g+B A},
$$

где $B$ - постоянная интегрирования, а $\lambda=k_{3}\left(c^{2}-1\right) / 2 A c_{2} c^{3}$. В случае, когда $k_{2}=0$ и $k_{1}=k_{3}$, в уравнении (3.24) переменные разделяются и решение имеет вид

$$
\widetilde{H}_{4}(g)=k_{1}\left[B_{1}\left(\frac{c_{2} c+g}{c_{2} c-g}\right)^{k_{1}\left(c^{2}-1\right) / 2 A c_{2} c^{3}} e^{k_{1} / g A c^{2}}-1\right]^{-1},
$$

где $B_{1}$ - ненулевая постоянная интегрирования. Тогда решение уравнений Больцмана (3.1) согласно $(3.18)$, где $u_{0}, \tilde{u}_{0}, v_{0}$ и $\tilde{v}_{0}$ заданы в $(3.3)$, а функция $\phi$ определена в $(3.17)$, имеет вид

$$
\begin{aligned}
u(t, x, y) & =\frac{c-1}{\sigma c^{2} A\left(c_{1}-c_{2} y\right)}\left(\widetilde{H}_{4}(g)+k_{1}\right), \\
\tilde{u}(t, x, y) & =\frac{c+1}{\sigma c^{2} A\left(c_{1}-c_{2} y\right)} \widetilde{H}_{4}(g), \\
v(t, x, y) & =\frac{c^{2}-1}{\sigma c^{2} A\left(c_{1}-c_{2} y+c_{2} c(x+t)\right)}\left(\widetilde{H}_{4}(g)+k_{1}\right), \\
\tilde{v}(t, x, y) & =\frac{c^{2}-1}{\sigma c^{2} A\left(c_{1}-c_{2} y-c_{2} c(x+t)\right)} \widetilde{H}_{4}(g) .
\end{aligned}
$$

Здесь $\widetilde{H}_{4}(g)$ задается в $(3.25)$, а $g-$ в $(3.14)$.

\section{4. ЗАКЛЮЧИТЕЛЬНЫЕ ЗАМЕЧАНИЯ}

Выше мы показали, что неявное решение уравнения Бейтмена можно эффективно использовать для решения как двумерных, так и трехмерных уравнений Больцмана. Основные результаты, сформулированные в предложениях 1 и 2, состоят в том, что большое число решений уравнений Больцмана можно построить, используя решения уравнений Риккати, так как последние линеаризуемы как обыкновенные дифференциальные 
уравнения второго порядка. Мы привели здесь лишь несколько примеров, чтобы продемонстрировать тип таких решений. Следует ожидать, что этот метод можно применить к другим нелинейным уравнениям в частных производных, для которых тест Пенлеве оказывается неэффективным. Однако наиболее важное свойство этой процедуры заключается в том, что она решает проблему условий совместимости на функцию $\phi$, которая связана с усечением нулевого уровня рядов Пенлеве. Для уравнений Больцмана, которые были рассмотрены в настоящей работе, мы смогли решить эту задачу при помоши решения уравнения Бейтмена. Этот шаг является сушественным, но пока не ясно, может ли уравнение Бейтмена давать интересные решения для других нелинейных уравнений в частных производных. Например, эволюционные уравнения, которые изучались Кариэлло и Табором [7], по-видимому, непосредственно не связаны с уравнением Бейтмена.

\section{Список литературы}

[1] J. Weiss, M. Tabor, G. Carnevale. J. Math. Phys. 1983. V. 24. P. 522-526.

[2] W.-H. Steeb, N. Euler. Nonlinear Evolution Equations and the Painlevé Test. Singapore: World Scientific, 1988.

[3] F. Cariello, M. Tabor. Physica D. 1989. V. 39. P. 77-94.

[4] N. Euler, O. Lindblom. Int. J. Diff. Equat. Appl. 2000. V. 1. P. 205-222.

[5] С. Л. Годунов, У. М. Султангазин. УМН. 1971. Т. 26. № 3. С. 3-51.

[6] H. Cabannes. Survey on exact solutions for discrete models of the Boltzmann equation. In: Computational Fluid Dynamics. Eds. D. Leutloff, R. C. Srivastava. Berlin: Springer, 1994. P. 103-114.

[7] F. Cariello, M. Tabor. Physica D. 1991. V. 53. P. 59-70.

Поступила в редакцию 2.VIII.2001 г. 